\title{
Current Indications for Tonsillectomy and Adenoidectomy
}

\author{
David A. Randall, MD
}

This paper reviews current indications for otolaryngology consultation for tonsillectomy and adenoidectomy (T\&A). Despite often being performed concurrently, these procedures should be considered separate surgeries done for different indications. The American Academy of Otolaryngology - Head and Neck Surgery published tonsillectomy guidelines for children in 2019. These recommendations are often extrapolated to adults in clinical practice despite less robust literature support for this age group. T\&A should be recommended for pediatric obstructive sleep apnea. Specific frequencies of tonsillitis have been identified that indicate benefit from tonsillectomy in normal children; certain modifying health factors warrant consideration of surgery with fewer infections. The guidelines include consideration of tonsillectomy for poorly validated indications such as halitosis, febrile seizure, dental malocclusion, dysphagia, dysphonia, and psoriasis. (J Am Board Fam Med 2020;33:1025-1030.)

Keywords: Adenoidectomy, Adenoids, Hypertrophy, Obstructive Sleep Apnea, Otolaryngology, Streptococcal Infections, Tonsillectomy, Tonsils

\section{Introduction}

Tonsillectomy and adenoidectomy remain common surgeries, yet their efficacy had not been well studied until 1984. The pediatric population has more robust literature than the adult population. Current pediatric guidelines support surgery for patients with obstructive sleep-disordered breathing (OSDB) and recurrent tonsillitis. These indications have, in clinical practice, been extrapolated to adults but this requires further research. The 2019 AAOHNS guidelines for pediatric tonsillectomy find that surgery may be appropriate with modifying factors such as multiple antibiotic allergy, certain syndromes, recurrent peritonsillar abscess, enuresis, or poorly validated indications such as halitosis, febrile seizure, malocclusion, dysphagia, or dysphonia. ${ }^{2}$ It should be stressed that tonsillectomy and adenoidectomy constitute different procedures with different indications. This article discusses indications for otolaryngology referral from primary care physicians.

This article was externally peer reviewed.

Submitted 26 January 2020; revised 21 April 2020; accepted 26 April 2020.

Funding: None.

Conflict of interest: None.

Corresponding author: David A. Randall, MD, Retired otolaryngologist, 851 Collier Court \#7, Marco Island, FL 34145 (E-mail: davidarandallmd@gmail.com).

\section{Recurrent Tonsillitis}

Recurrent tonsillitis in children and adults remains a common indication for otolaryngology consultation. Several studies have demonstrated modest benefit for tonsillectomy in children having recurrent tonsillitis. ${ }^{2,10}$ Concurrent adenoidectomy conferred no additional benefit. ${ }^{11}$ One article demonstrated fewer throat infections for 2 years after surgery. ${ }^{10}$ A Cochrane Review pooled this and other pediatric data and found 1 year of benefit. Study heterogeneity and patient numbers have limited the strength of these studies. Parental satisfaction with tonsillectomy, however, is as high as $92 \% .12$ Current American Academy of Otolaryngology-Head and Neck Surgery guidelines (AAOHNS guidelines) advise that children with 7 bouts of tonsillitis in 1 year, 5 annual bouts for 2 years, or 3 annual bouts for 3 years may benefit from tonsillectomy while those having fewer bouts will not. ${ }^{2,13,14}$

Tonsillectomy is also reasonable with fewer infections but with modifying circumstances. Some children have unusually severe symptoms or require hospitalization. Others factors include multiple antibiotic allergy, recurrent peritonsillar abscess, and periodic fever, aphthous ulcers, pharyngitis, cervical adenopathy (PFAPA) syndrome, internal jugular vein thrombosis (Lemierre's syndrome) due to tonsillitis, and rheumatic heart disease. Poorly validated indications include chronic tonsillitis, persistent group- 


\begin{tabular}{lc}
\hline Recommendation & Strength \\
\hline Referral for tonsillectomy is appropriate for children having 7 bouts of & SORT: A \\
tonsillitis in 12 months, 5 annual bouts for 2 years, or 3 annual bouts for 3 & \\
years. Watchful waiting is indicated for lesser frequency. & SORT: B \\
Tonsillectomy may reasonably be offered to children having fewer & SORT: A \\
infections given certain modifying factors. & SORT: B \\
Tonsillectomy or adenotonsillectomy should be recommended for pediatric & SORT: C \\
obstructive sleep - disordered breathing felt due to adenoid or tonsillar & \\
hypertrophy. & SORT: B \\
Tonsillectomy has shown benefit for adults with recurrent tonsillitis. & \\
Tonsillectomy/adenotonsillectomy may be indicated in adults with sleep & SORT: C \\
disordered breathing and tonsil hypertrophy. & \\
Adenoidectomy may be considered for chronic middle ear effusion and & \\
recurrent otitis media in children. & \\
Tonsillectomy may be considered for poorly validated indications such as & \\
chronic tonsillitis, febrile seizures, muffled speech, halitosis, malocclusion, & \\
tonsillar hypertrophy, cryptic tonsils, and chronic pharyngeal carriage of & \\
group A $\beta$ hemolytic Streptococcus (GABHS). &
\end{tabular}

SORT, Strength of Recommendation Taxonomy

A $\beta$ hemolytic strep (GABHS) carriage, febrile seizure, halitosis, tonsillitis, dysphonia, dysphagia, and malocclusion. There is a substantial role in shared decision making for this last group of problems. These situations warrant consideration of referral to an otolaryngologist. There is a substantial role for shared decision making between the caregivers and the surgeon. ${ }^{2}$

A relative paucity of literature exists for adults. Two studies reported short-term benefit from tonsillectomy for recurrent pharyngitis. One demonstrated significantly fewer bouts of GABHS and days with sore throat in the first 90 days after tonsillectomy. ${ }^{3}$ The other found that its surgical group had fewer bouts of pharyngitis and also decreased absenteeism from work or school. ${ }^{4}$ A Cochrane Review combined these data and found that surgery conferred 3.6 fewer cases of tonsillitis and 10.6 less sore throat days for 6 months postoperatively. However, they judged this information to be of lower quality due to the short period studied and statistical heterogeneity. ${ }^{10}$ It is presently a common and quite reasonable clinical practice to offer otolaryngology evaluation for tonsillectomy in adults meeting the pediatric criteria.

\section{Recurrent Peritonsillar Abscess}

Peritonsillar abscess (PTA) develops between the tonsil and pharyngeal constrictors, and commonly occurs in adolescents and young adults. ${ }^{15}$ Ten to $15 \%$ of patients-typically under 40 years of age or with recurrent tonsillitis-have 1 or more PTAs. ${ }^{15,16}$ AAOHNS guidelines follow evidence-based common practice listing recurrent PTA as an indication of tonsillectomy. ${ }^{2}$ It is also common practice in adults and has literature support. ${ }^{15}$ Patients may undergo tonsillectomy during an acute abscess, known as a "hot" or "Quinsy" tonsillectomy. In patients meeting criteria for tonsil removal, this is a useful technique for children or uncooperative patients.

\section{Pediatric OSDB}

OSDB occurs in $1.2 \%$ to $5.7 \%$ of children. ${ }^{2}$ Associated sequelae include cardiopulmonary disease and growth impairment. ${ }^{17}$ Memory problems, poor school performance, as well as behavioral issues such as aggression, attention deficit, hyperactivity, and aggression have been associated with this condition. ${ }^{2,18}$ Hypertrophy of the adenoid, and particularly, the tonsils are the most frequent cause. ${ }^{2}$ The combined volume of these lymphatic structures tends to correlate with severity. Other potential etiologies include obesity, Down syndrome, craniofacial abnormalities, and neuromuscular disease. ${ }^{2}$

Diagnosis of obstructive sleep-disordered breathing (ODSB) in children is often based on history and examination but may also include video and audio taping, overnight pulse oximetry, sleep study, or validated sleep questionnaires. ${ }^{2,17,19}$ Current AAOHNS guidelines recommend polysomnography in children below the age of 2 years or if they 
have obesity, Down syndrome, craniofacial abnormalities, neuromuscular disorder, sickle-cell disease, or mucopolysaccharidoses. ${ }^{2}$ Certain patients are at higher risk for persistent OSDB after tonsillectomy or adenotonsillectomy - those with asthma, age over 7 years, obesity, or more severe sleep apnea-but surgery remains a viable option. ${ }^{2}$

Meta-analysis has demonstrated benefit from adenotonsillectomy for pediatric OSDB although some studies are of lesser quality. ${ }^{17,19}$ Quality-oflife scores improved for at least 2 years following surgery. ${ }^{2}$ Meta-analysis also showed significant benefit with regard to sleep disturbance, physical suffering, emotional distress, daytime problems, and caregiver concerns. ${ }^{17}$ Children with OSDB should be referred for evaluation for tonsillectomy or adenotonsillectomy. ${ }^{2}$

\section{Enuresis}

Secondary nocturnal enuresis is defined as bed wetting after a 6-month dry period. It is associated with and is a strong predictor of OSDB. ${ }^{2,20} \mathrm{~A}$ review of 7 studies on children with OSDB showed a prevalence of nocturnal enuresis of $31 \%$. This was reduced to $16 \%$ after adenotonsillectomy. ${ }^{20}$ Tonsil/ adenoid removal remains an effective option for children with enuresis and OSDB and these patients should receive an otolaryngology evaluation. ${ }^{2}$ ]

\section{Adult Obstructive Sleep Apnea}

Two to $4 \%$ of adults have OSDB. ${ }^{5}$ Positive airway pressure pneumatically stents the upper airway and constitutes primary treatment for adult OSDB. ${ }^{5}$ Oral mandibular-advancement splints may be considered as well, although these can aggravate temporomandibular joint conditions. Referral for surgical management as initial therapy may be considered with obvious obstructive anatomy (such as tonsil hyperplasia). Positive airway pressure remains the mainstay of therapy. Tonsillectomy/adenoidectomy are often reserved supplement ODSB that incompletely responds to Papanicolaou or for patients unable to tolerate airway pressure therapy. ${ }^{5}$

\section{Suspicion of Malignancy}

Unilateral tonsillar enlargement may indicate neoplasm, typically Hodgkin's lymphoma in children and young adults and squamous cell carcinoma in the older population. Squamous cell carcinoma has long been associated with tobacco and alcohol use, and is now linked with human papilloma virus, particularly in younger patients. Recent tonsil enlargement, a suspicious tonsil lesion, or persistent cervical adenopathy may suggest tonsillar malignancy. Rather surprisingly, visible tonsillar asymmetry actually correlates poorly with true size difference in children. ${ }^{21}$ Tonsillectomy is also indicated when the primary site of a head and neck malignancy is unknown. ${ }^{22}$ Patients typically present with enlarging cervical lymph nodes that suggest metastatic disease based on fine-needle aspiration cytology. The tonsil ipsilateral to the adenopathy is removed at the time of direct laryngoscopy evaluation with its standard directed site biopsies. ${ }^{22}$

\section{Malocclusion}

Upper airway obstruction in children seems linked to altered dentofacial development. ${ }^{23,24}$ Habitual mouth breathing from adenotonsillar hypertrophy may affect the relative position of the jaw and tongue, thus altering lingual pressure on dental structures and affecting growth. ${ }^{24}$ Dentists may request consideration of surgical management (usually for adenoidal obstruction) and may also recommend orthodontia and palatal expanders. Two recent meta-analyses found trends toward improvement and normalization of dental arch morphology following adenoidectomy and/or tonsillectomy. ${ }^{23,24}$ Malocclusion is considered a poorly validated but potential indication for surgery in children. ${ }^{2}$ Otolaryngology consultation for this more commonly originates from dental colleagues.

\section{Alteration in Speech and Swallowing}

Tonsillar, and less often, adenoid adenoidal hypertrophy may cause dysphagia-typically for solids. Adenotonsillar size and obstruction may also cause a hyponasal voice or interfere with speech. Dysphagia and failure to thrive associated with this has been considered an indication for tonsillectomy and adenotonsillectomy. ${ }^{25}$ Involvement of a speech therapist in evaluation would be prudent in the interest of considering all noninvasive options before proceeding to a surgical procedure for dysphonia or dysphagia. In practice, this is likely a less common indication for referral from the primary care physician.

\section{Otitis Media with Effusion}

Otitis media with effusion (OME) may follow upper respiratory infection or acute otitis media 
(AOM); it may also occur due to Eustachian tube dysfunction. OME is most common between the ages of 6 months and 4 years, particularly in children with Down syndrome or cleft palate. ${ }^{2}$ It may be associated with hearing loss, imbalance, school or behavioral issues, otalgia, and $\mathrm{AOM}^{2}{ }^{2}$ Surgical intervention is indicated after 3 months of OME associated with hearing loss but may considered sooner in "atrisk," children who have hearing, speech, cognitive, sensory, or behavioral conditions. ${ }^{2}$ Although modest benefit is conferred by performing adenoidectomy in addition to tympanostomy tube (TT) placement, it has been customary to initiate treatment of persistent OME with tubes alone. ${ }^{8}$ Current AAOHNS guidelines recommend TT for children under the age of 4 in the absence of separate indication for adenoidectomy such as nasal obstruction or the rare case of adenoiditis. For older children, adenoidectomy may be offered along with TT for persistent OME. ${ }^{2}$

\section{Recurrent AOM}

Adenoidectomy has long been used as a treatment for recurrent AOM. ${ }^{9}$ Mechanisms of the adenoid causing eustachian tube dysfunction as well as its being a bacterial reservoir have been posited to explain the efficacy of adenoidectomy for this condition. ${ }^{7}$ Meta-analysis has shown benefit with adenoidectomy in reducing the need for additional TT placement and TT otorrhea. ${ }^{2,8,9,26}$ For recurrent AOM persistent after TT placement, it is common and appropriate to offer adenoidectomy with a second set of TT.

\section{Pediatric Rhinosinusitis and Nasal Obstruction}

Chronic pediatric rhinosinusitis (RS) has been variably defined as greater than 90 days of RS symptoms along with either endoscopic or radiographic findings. ${ }^{27}$ Children may manifest cough, nasal congestion, rhinorrhea, irritability, or behavioral problems; headache is less common. ${ }^{28}$ Disease severity seems unrelated to adenoid size. ${ }^{27}$ Adenoidal harboring of bacteria as well as biofilms and exotoxins have been implicated etiologies. ${ }^{27,28}$ Meta-analysis has shown a $50 \%$ to $84 \%$ improvement after adenoidectomy in younger patients but the benefit of the procedure is with a less clear role in preadolescents. $^{27,28}$ Otolaryngology referral for consideration of adenoidectomy is recommended for refractory pediatric RS. ${ }^{28}$

Adenoid removal for hypertrophy has been performed for nasal obstruction recalcitrant to medical therapy. However, this procedure does involve the risks of general anesthesia as well as potential adverse effects such as velopharyngeal insufficiency or nasopharyngeal stenosis. The literature seems to support adenoidectomy mostly in conjunction with treatment of other medical conditions such as sinusitis and OSDB.

\section{PFAPA Syndrome}

The syndrome of periodic fever, aphthous ulcers, pharyngitis, and cervical adenopathy (PFAPA) is a rare but recognized entity. It typically affects children under age 5 years and involves bouts of 5 days or less, approximately every 3 to 6 weeks. Steroids provide rapid resolution but shorten time between episodes. ${ }^{2}$ A Cochrane Review found tonsillectomy reduced recurrence frequency by a factor of 10 and lessened the average duration from 3.5 to 1.8 days. ${ }^{29}$ The AAOHNS guidelines consider otolaryngology and referral and tonsillectomy as an option for PFAPA. $^{1,29}$

\section{Psoriasis}

Psoriasis affects $1 \%$ to $3 \%$ of the population and is felt to be T-cell mediated. ${ }^{30,31}$ Exacerbations have regularly been noted with pharyngitis due to GABHS expressing surface $M$ protein. ${ }^{30-32}$ Its proposed mechanism is a cross-reaction between GABHS antigens and similar skin proteins. ${ }^{30,31}$ The literature largely comprises uncontrolled case series but indicates tonsillectomy to be an effective treatment. $^{30,31}$ One randomized investigation of adults demonstrated benefit for at least 2 years. This showed a statistically significant reduction in the Psoriasis Area and Severity Index score. Improvement also correlated with a reduction of circulating effector $\mathrm{T}$ cells. ${ }^{32}$ The primary care physician should consider otolaryngology referral for possible tonsillectomy for patients with psoriasis refractory to medical management.

\section{Halitosis}

Tonsillectomy has been considered a relative indication for persistent halitosis despite absence of controlled study. ${ }^{2,3}$ Halitosis primarily originates 
from an oral source such as tongue coating or dental disease. A necrotic upper airway malignancy represents a possible but rare cause. ${ }^{33}$ Bad breath may also be caused by tonsillar crypt debris. ${ }^{25,34}$ A number of case series of tonsillectomy and tonsil crypt ablation have reported halitosis improvement or resolution in $75 \%$ to $98 \%$ of cases. ${ }^{34}$ The crypt procedures offer potential advantages of reduced convalescent time and pain and avoidance of general anesthesia. Unfortunately, these require repeated treatments; 1 report averaged $7 . .^{34}$ Tonsillectomy, despite its postoperative morbidity, offers a 1-time therapy and may be the preference of some patients. Despite the paucity of data, tonsillectomy or crypt ablation are reasonable options for socially bothersome halitosis after excluding primary oral sources. ${ }^{2,33,34}$

\section{Conclusions}

Tonsillectomy and adenoidectomy have demonstrated benefit for a number of conditions described above. The AAOHNS guidelines more strongly recommend surgery more strongly for some medical conditions (eg, pediatric OSDB) than for others. Satisfaction for tonsillectomy for tonsillitis remains high. ${ }^{2,12}$ The role for shared decision making is more apparent with less well or poorly validated issues (eg, halitosis) than others. Further study is warrantedparticularly in adult populations-to better understand the benefits and risk of surgical intervention for these conditions. Until then, primary care physicians may use the AAOHNS guidelines to make prudent decisions regarding evaluation and treatment options to pursue before otolaryngology referral.

To see this article online, please go to: http://jabfm.org/content/ 33/6/1025.full.

\section{References}

1. Ebell M, Siwek J, Weiss B, et al. Strength of Recommendation Taxonomy (SORT): A patientcentered approach to grading evidence in the medical literature. J Am Board Fam Pract 2004;17:59-67.

2. Mitchel R, Archer S, Ishman S, et al. Clinical practice guideline: tonsillectomy in children (update). Otolaryngol Head Neck Surg 2019;160:S1-S42.

3. Alho OP, Koivunen P, Penna T, Teppo H, Koskela $M$, Luotonen J. Tonsillectomy versus watchful waiting in recurrent streptococcal pharyngitis in adults: randomized controlled trial. $\mathrm{Br}$ Med J 2007;334:939-44.

4. Koskenkorva T, Koivunen P, Koskela M, Niemela O, Kristo A, Alho O. Short-term outcomes of tonsillectomy in adult patients with recurrent pharyngitis: a randomized controlled trial. Can Med Assoc J 2013;14:E331-E336.

5. Epstein LJ, Kristo D, Strollo PJ, et al. Clinical guideline for the evaluation, management, and long-term care of obstructive sleep apnea in adults. J Clin Sleep Med 2009;5:263-76.

5. Heidemann CH, Lous J, Berg J, et al. Danish guidelines on management of otitis media in preschool children. Int J Pediatr Otorhinolaryngol 2016;87:154-63.

7. Gisselsson-Solen M. Acute otitis media in children -Current treatment and prevention. Curr Infec Dis Rep 2015;17:22-8.

8. Mikals S, Brigger M. Adenoidectomy as adjuvant to primary tympanostomy tube placement a systematic review and meta-analysis. JAMA Otolaryngol Head Neck Surg 2014;140:95-101.

9. Granath A. Recurrent otitis media: what are the options for treatment and prevention? Curr Otorhinolaryngol Rep 2017;5:93-100.

10. Burton M, Glasziou P, Chong L, Venekamp R. Tonsillectomy or adenotonsillectomy versus nonsurgical treatment for chronic/recurrent tonsillitis. Cochrane Database System Rev 2014;1:1-62.

11. Paradise J, Bluestone C, Bachman R, et al. Efficacy of tonsillectomy for recurrent throat infection in severely affected children. N Engl J Med 1984;310:674-83.

12. Barraclough J, Anari S. Tonsillectomy for recurrent sore throats in children: indications, outcomes, and efficacy. Otolaryngol Head Neck Surg 2014;150:722-9.

13. Paradise J, Bluestone C, Colborn D, Bernard B, Rockett H, Kurs-Lasky M. Tonsillectomy and adenotonsillectomy for recurrent throat infection in moderately affected children. Pediatrics 2002;110:7-15.

14. van Staaij BK, van den Akker EH, Rovers MM, Hordijk GJ, Hoes AW, Schilder AGM. Effectiveness of adenotonsillectomy in children with mild symptoms of throat infections or adenotonsillar hypertrophy: open, randomized trial. Br Med J 2004;329:651-6.

15. Johnson R, Stewart M, Wright C. An evidence-based review of the treatment of peritonsillar abscess. Otolaryngol Head Neck Surg 2003;128:332-43.

16. Herzon F. Peritonsillar abscess: incidence, current management practices, and a proposal for treatment guidelines. Laryngoscope 1995;105:1-17.

17. Baldassari C, Mitchell R, Schubert C, Rudnick E. Pediatric obstructive sleep apnea and quality of life: a meta-analysis. Otolaryngol Head Neck Surg 2008;138:265-73.

18. Patel H, Straight C, Lehman E, Tanner M, Carr M. Indications for tonsillectomy: a 10 -year retrospective review. Int J Pediatr Otorhinolaryngol 2014;78:2151-5.

19. Todd C, Bareiss A, McCoul E, Rodriguez K. Adenotonsillectomy for obstructive sleep apnea and quality of life: systematic review and meta-analysis. Otolaryngol Head Neck Surg 2017;157:767-73. 
20. Jeyakumar A, Rahman S, Armbrecht E, Mitchell R. The association between sleep-disordered breathing and enuresis in children. Laryngoscope 2012; 122:1873-7.

21. Van Lierop A, Prescott C, Fagan J, Sinclair-Smith C. Is diagnostic tonsillectomy indicated in all children with asymmetrically enlarged tonsils? S Afr Med J 2007;97:367-70.

22. Randall D, Johnstone P, Foss R, Martin P. Tonsillectomy in diagnosis of the unknown primary of the head and neck. Otolaryngol Head Neck Surg 2000;122:52-5.

23. Zhu Y, Li J, Tang Y, et al. Dental arch dimensional changes after adenoidectomy or tonsillectomy in children with airway obstruction a meta-analysis and systematic review under PRISMA guidelines. Medicine 2016;95:e4976.

24. Becking BE, Verweij JP, Kalf-Scholte SM, Valkenburg C, Bakker EW, van Merkesteyn JR. Impact of adenotonsillectomy on the dentofacial development of obstructed children: a systematic review and metaanalysis. Eur J Orthod 2017;39:509-17.

25. Darrow DH, Siemens C. Indications for tonsillectomy and adenoidectomy. Laryngoscope 2002;112:6-10.

26. Schilder AGM, Marom T, Bhutta MF, et al. Otitis media: treatment and complications. Otolaryngol Head Neck Surg 2017;156:S88-S105.
27. Neff L, Adil E. What is the role of the adenoid in pediatric chronic rhinosinusitis? Laryngoscope 2015;125: 1282-3.

28. Magit A. Pediatric rhinosinusitis. Otolaryngol Clin North Am 2014;47:733-46.

29. Burton M, Pollard A, Ramsden J, Chong L, Venekamp R. Tonsillectomy for periodic fever, aphthous stomatitis, pharyngitis and cervical adenitis syndrome (PFAPA). Cochrane Database Syst Rev 2014;9:1-30.

30. Sigurdardottir SL, Thorleifsdottir RH, Valdimarsson $\mathrm{H}$, Johnston A. The role of the palatine tonsils in the pathogenesis and treatment of psoriasis. Br J Dermatol 2013;168:237-42.

31. Wu W, Debbaneh M, Moslehi H, Koo J, Liao W. Tonsillectomy as a treatment for psoriasis: a review. J Dermatolog Treat 2014;25:482-6.

32. Thorleifsdottir RH, Sigurdardottir SL, Sigurgeirsson $\mathrm{B}$, et al. Improvement of psoriasis is associated with a decrease in the frequency of circulating $T$ cells that recognize streptococcal determinants and homologous skin determinants. J Immunol 2012;188:5160-5.

33. Van den Broek A, Feenstra L, Baat C. A review of the current literature on management of halitosis. Oral Dis 2007;14:30-9.

34. Ferguson M, Aydin MM. Halitosis and the tonsils: a review of management. Otolaryngol Head Neck Surg 2014;151:567-74. 